\title{
An evaluation of the World Health Organization's 1997 and 2009 dengue classifications in hospitalized dengue patients in Malaysia
}

\author{
Zuraihan Zakaria ${ }^{1}$, Nur A Zainordin², Benedict LH Sim ${ }^{3}$, Masliza Zaid ${ }^{3}$, Umi S Haridan ${ }^{1}$, Abu TA Aziz ${ }^{1}$, \\ Rafidah H Shueb ${ }^{4}$, Mahiran Mustafa ${ }^{5}$, Nik KN Yusoff ${ }^{5}$, Alam S Malik ${ }^{6}$, Christopher KC Lee ${ }^{3}$, Sazaly \\ Abubakar $^{7,8}$, Boon Peng Hoh ${ }^{1}$ \\ ${ }^{1}$ Institute of Medical Molecular Biotechnology (IMMB), Faculty of Medicine, Universiti Teknologi MARA, Sungai \\ Buloh, Selangor, Malaysia \\ ${ }^{2}$ Faculty of Medicine, Universiti Teknologi MARA, Sungai Buloh, Selangor, Malaysia \\ ${ }^{3}$ Hospital Sungai Buloh, Sungai Buloh, Selangor, Malaysia \\ ${ }^{4}$ Department of Medical Microbiology and Parasitology, School of Medical Sciences, Health Campus, Universiti \\ Sains Malaysia, Kelantan, Malaysia \\ ${ }^{5}$ Hospital Raja Perempuan Zainab II, Kelantan, Malaysia \\ ${ }^{6}$ Faculty of Medicine, Quest International University Perak (QIUP) Perak Darul Ridzuan, Malaysia \\ ${ }^{7}$ Department of Medical Microbiology, Faculty of Medicine, Universiti Malaya, Kuala Lumpur, Malaysia \\ ${ }^{8}$ Tropical Infectious Disease Research and Education Centre, Faculty of Medicine, Universiti Malaya, Kuala \\ Lumpur, Malaysia
}

\begin{abstract}
Introduction: The latest revised version of the World Health Organization's dengue classification was released in 2009. A handful of studies have taken initiatives to evaluate the old and revised guidelines to determine early signs and symptoms of severe dengue. This retrospective study aimed to compare the classification of dengue using both the 1997 and 2009 guidelines in a selected cohort of dengue patients from Peninsular Malaysia between 2008 and 2012.

Methodology: Adult dengue patients were recruited from tertiary hospitals in two different states, Selangor and Kelantan, in Peninsular Malaysia. Their clinical manifestations were assessed.

Results: A total of 281 confirmed dengue patients were enrolled; the mean duration of illness at admission was five days. Of these, $88.6 \%$, $10.7 \%$, and $0.7 \%$ were classified according to the 1997 guidelines as having dengue fever (DF), dengue hemorrhagic fever (DHF), and dengue shock syndrome (DSS), respectively. When the WHO 2009 guidelines were applied, 17.1\%, 78.3\%, and 4.6\% were classified as dengue without warning signs, dengue with warning signs, and severe dengue, respectively.

Conclusions: Our data suggests that the revised WHO 2009 guidelines stratify a much larger proportion of patients into a category that requires a higher level of medical and nursing care.
\end{abstract}

Key words: dengue; WHO 1997; WHO 2009; Malaysia

J Infect Dev Ctries 2014; 8(7):869-875. doi:10.3855/jidc.4283

(Received 06 October 2013 - Accepted 05 February 2014)

Copyright (C 2014 Zakaria et al. This is an open-access article distributed under the Creative Commons Attribution License, which permits unrestricted use, distribution, and reproduction in any medium, provided the original work is properly cited.

\section{Introduction}

Dengue is the most prevalent mosquito-borne viral disease affecting people in the tropic and sub-tropic regions of the world, caused by the infection of dengue virus (DENV). The virus exists as four related but antigenically distinct serotypes, namely DENV 1-4. These viruses are transmitted to humans via infected mosquitoes, namely Aedes sp. Individuals infected with any DENV serotype may remain asymptomatic or may present with symptoms ranging from mild febrile illness to severe and occasionally fatal hemorrhagic disease.
Globally, dengue fever and severe dengue cases have risen from less than 1,000 cases in 1955 to almost $1,000,000$ cases in 2007. The number of countries reporting dengue cases has also increased to more than 60 countries [1]. Recently, Bhatt et al. [2] estimated an alarming 390 million dengue infection worldwide per year, which is three times higher than predicted by the World Health Organization (WHO). In Malaysia, the number of reported dengue cases has also shown an upward trend, with around 6,500 cases in 1995 to 32,767 cases in 2002 and more than 49,000 
cases in 2007 [3]. All four DENV serotypes have been co-circulating in Malaysia [4].

Under the earlier WHO classification [5], patients infected with dengue were either classified as having dengue fever (DF) or dengue hemorrhagic fever (DHF) (Table 1). DHF patients were further stratified into four categories depending on severity, namely DHF-I, II, III, and IV. DHF-III and IV are defined as dengue shock syndrome (DSS) when patients manifest signs of circulatory failure. However, there were problems with this classification, as at times it proved difficult to apply in the clinical context. Furthermore,

Table 1. The old and new WHO dengue classifications (extracted from WHO 1997 and 2009)

\section{Dengue Fever (DF)}

\section{WHO 1997 dengue classifications}

Acute febrile illness with two or more of the following:

- Headache

- Retro-orbital pain

- Myalgia

- Leukopenia

- Anthralgia

- Rash

- Hemorrhagic manifestations

- Supportive serology or occurrence at the same location as other confirmed cases of dengue fever

Dengue Hemorrhagic Fever (DHF)

All of the following must be present:

- Fever or history of acute fever, lasting 1-7 days, occasionally biphasic

- Hemorrhagic manifestations:

-Positive torniquet test;

-Petechia, equimosis, purpura or bleeding from mucosa, gastrointestinal tract, injection sites or other locations; or

-Haematemesis/melena

- Thrombocytopenia $\left(<100,000\right.$ platelets per $\left.\mathrm{mm}^{3}\right)$

- $\quad$ Evidence of plasma leakage due to increased vascular permeability

Dengue Shock Syndrome (DSS)

DHF with hypotension for age or narrow pulse pressure $(<20 \mathrm{mmHg})$, plus one of the following:

- $\quad$ Rapid and weak pulse

- Cold, clammy skin, restlessness

Dengue without warning signs

Fever and two of the following:

- Nausea, vomiting

- Rash

- Aches and pains

- Leukopenia

- Positive tourniquet test

Dengue with warning signs

Dengue as defined above with any of the following:

- Abdominal pain or tenderness

- Persistent vomiting

- Clinical fluid accumulation

- Mucosal bleeding

- Lethargy, restlessness

- Liver enlargement $>2 \mathrm{~cm}$

- Laboratory: increase in HCT concurrent with rapid decrease in platelet count

Severe dengue

Dengue with at least one of the following criteria:

- $\quad$ Severe plasma leakage leading to:

-shock (DSS)

-fluid accumulation with respiratory distress

- $\quad$ Severe bleeding as evaluated by clinician

- Severe organ involvement

-liver: AST or ALT $\geq 1000$

-CNS: impaired consciousness

-failure of heart and other organ

\section{WHO 2009 dengue classifications}


there were patients with severe dengue manifestations that did not fulfil the criteria of DHF [6,7]. This was also seen in Malaysia [8,9]. Thus, this classification underwent a proposed revision by the WHO in 2009, when the DHF criteria was revised and replaced with dengue with or without warning signs and severe dengue (Table 1).

Several reports have evaluated the differences between the two classification schemes. A study conducted in 18 countries, of which Malaysia was one, showed that a higher percentage of dengue cases could not be classified when the WHO 1997 classification was applied, as compared to the revised classification [10]. Similarly, all the fatal cases during a five-year study in Singapore fulfilled the severe dengue criteria using the WHO 2009 classification, but only 36\% fulfilled the same criteria using the WHO 1997 classification [11]. On the other hand, another study indicated that both definitions are sensitive, but the diagnosis of dengue becomes more challenging in elderly patients [12], suggesting that there are still limitations to both guidelines. Hence, a conclusion could not be drawn about its applicability.

Therefore, this study aimed to evaluate both classifications in identifying severe dengue cases in Malaysia.

\section{Methodology}

Ethics approval

The study was approved by the research and ethics committees of Universiti Sains Malaysia (USM) [(USMKK/PPP/JEPeM (211.3[6])], Universiti Teknologi MARA (UiTM) (600-RMI [5/1/6]), and the Malaysian Ministry of Health (MOH) (NMRR-091128-4211).

\section{Sample collection}

Four hundred and thirty-nine hospitalized suspected adult dengue patients, 13 years of age or older were recruited from three different hospitals, namely Hospital Universiti Sains Malaysia (HUSM), Hospital Raja Perempuan Zainab II (HKB), and Hospital Sungai Buloh (HSB). Among these, 185 confirmed dengue patients were from HUSM and HKB and were recruited between 2008 and 2010. Both hospitals are located in the state of Kelantan. Another 96 patients were confirmed dengue patients from HSB (recruited between 2010 and 2012), located in the state of Selangor. Blood samples were collected on the first day of admission, and the clinical data were retrieved from standardized case report forms after the patients were discharged. Laboratory confirmation, namely an
IgM serological test, was carried out at the respective hospitals. These tests were independently repeated at least twice to confirm the results in addition to $\mathrm{IgG}$ serological tests. Dengue-specific IgG and IgM enzyme-linked immunosorbent assay (ELISA) kits (PanBio Diagnostics, Brisbane, Australia) were used in our laboratory. Diagnosis of dengue was determined by the expert clinicians at each study centre, respectively, based on the WHO criteria $[1,5]$.

\section{Inclusion and exclusion criteria}

Informed consent was collected from all recruited patients upon hospitalization. Clinically diagnosed patients with positive serological test either for IgG, IgM, or both were included in this study. Patients who had been co-infected with other pathogens, or were negative for both IgG and IgM, were excluded.

\section{WHO classifications [1,5]}

Table 1 shows the previous and recent WHO classifications for dengue severity $[1,5]$. According to the Malaysian Ministry of Health [3], the increase of hematocrit (Hct) was defined as more than $46 \%$ and $40 \%$ for males and females, respectively. This is based on the local normal range of Hct in adults and is due to the unavailability of baseline Hct levels in the respective study centers.

\section{Statistical analysis}

Chi-square was used to evaluate statistical differences in categorical variables between groups. The Statistical Package for Social Sciences (SPSS) version 20 (SPSS Inc., Chicago, IL, USA) was used for data analyses.

\section{Results}

Among 439 patients, 281 (64\%) dengue subjects were confirmed via serological tests (IgM and/or IgG). The excluded subjects were either negative for the serological tests, diagnosed to have co-infection during admission, or their clinical data were unavailable.

Table 2 shows the demographic data of the recruited subjects. Males comprised $60 \%$ of the subjects $(\mathrm{p}=0.001)$, and more than $80 \%$ of the subjects were under 50 years of age. A summary of clinical data presentation with prevalence is shown in Table 3. 
Table 2. Demographic data

\begin{tabular}{|c|c|c|c|}
\hline & & Number & $\%$ \\
\hline \multirow{4}{*}{ Race } & Malay & 236 & 83.69 \\
\hline & Indian & 11 & 3.90 \\
\hline & Other Malaysians & 4 & 1.42 \\
\hline & Non-Malaysians & 3 & 1.06 \\
\hline \multirow[b]{5}{*}{ Age group (years) } & $13-20$ & 59 & 20.92 \\
\hline & $21-30$ & 85 & 30.14 \\
\hline & $31-40$ & 44 & 15.6 \\
\hline & $41-50$ & 47 & 16.67 \\
\hline & $51-60$ & 23 & 8.16 \\
\hline
\end{tabular}

Data shows the distribution of gender, race, and age of subjects involved in this study ( $\mathrm{n}=281)$. Other Malaysians: Siamese, Orang Asli and East Malaysians. Non-Malaysians: Bangladeshi, Indonesian and Pakistani. N/A: not available. ${ }^{*} \mathrm{p}=0.001$.

Table 3. Clinical data presentation with prevalence

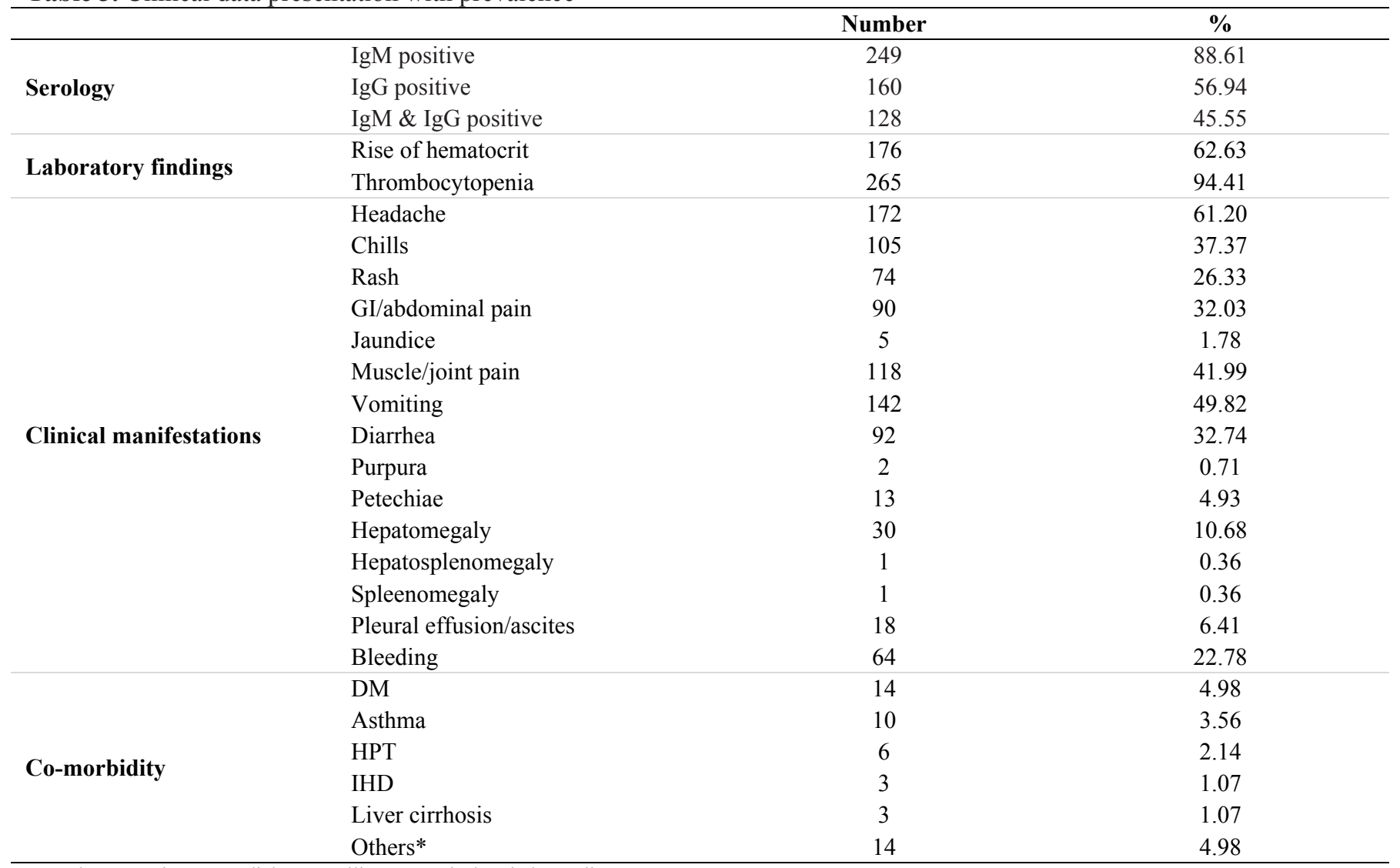

HPT: hypertension, DM: diabetes mellitus, IHD: ischemic heart disease

*Kidney failure, hepatitis, transaminitis, fatty liver, hyperlipidaemia, gastric and gout

Bleeding manifestations: epistaxis, haemoptysis, haematemesis, maleana, fresh rectal bleeding, haematuria, vaginal bleed, bleeding under the skin such as petechiae or purpura, or skin bruises and bleeding from the ear 
When patients were classified according to the WHO 1997 guidelines, DF was found most frequently (88.6\% of the cases), followed by DHF $(10.7 \%)$ and DSS $(0.7 \%)$ (Figure 1$)$. The trend shifted when they were classified based on the WHO 2009 guidelines, whereby the majority of the patients were classified as dengue with warning signs (DW) (78.29\%) (Figure 2).

In the 1997 classification, DSS was considered the severest form of dengue. Patients who were classified in this category expressed bleeding manifestations, thrombocytopenia, and pleural effusion and/or ascites. However, in the 2009 classification, only $67 \%$ of patients with severe dengue (SD) had bleeding manifestations, 95\% had thrombocytopenia, and only $57 \%$ had pleural effusion and /or ascites. Bleeding

Figure 1. Classification of study subjects according to WHO 1997 dengue guidelines.

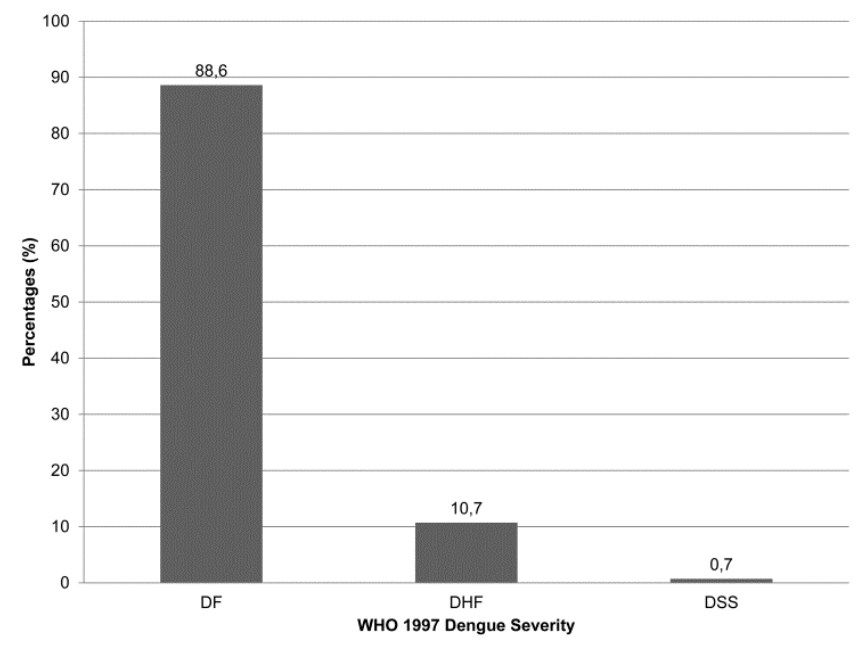

DF: dengue fever, DHF: dengue hemorrhagic fever, DSS: dengue shock syndrome. manifestations were included either by patients reporting or by detection of bleeding during their hospitalization. Pleural effusion and ascites were clinical findings made by the managing physician.

As shown in Table 4, $197(70 \%)$ of the patients classified as DF by WHO 1997 were classified as DW, $48(17 \%)$ as dengue without warning signs (D), and around $4(1 \%)$ as SD when grouped by WHO 2009. Not all patients grouped under SD by WHO 2009 were classified as DSS by WHO 1997; in fact, about 1.6\% of the DF patients (under WHO 1997 guidelines) were classified as SD. These were patients who had only hematemesis or increased AST as signs of severity and were not sufficient to be classified as DHF or DSS (Table 4).

Figure 2. Classification of study subjects according to WHO 2009 dengue guidelines.

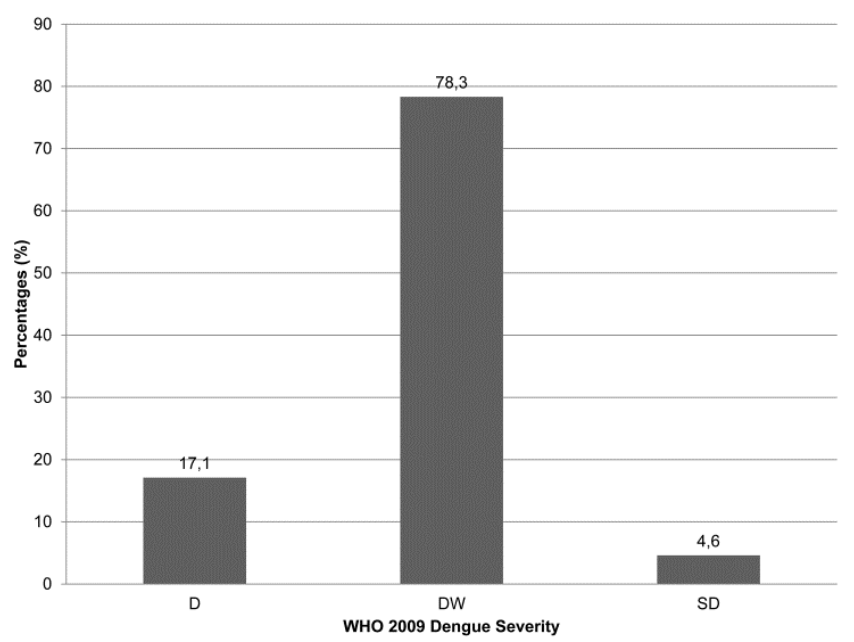

D: DF without warning signs, DW: dengue with warning signs, SD: severe dengue

Table 4. Concordance distribution of the study subjects according to WHO 2009 and WHO 1997 guidelines

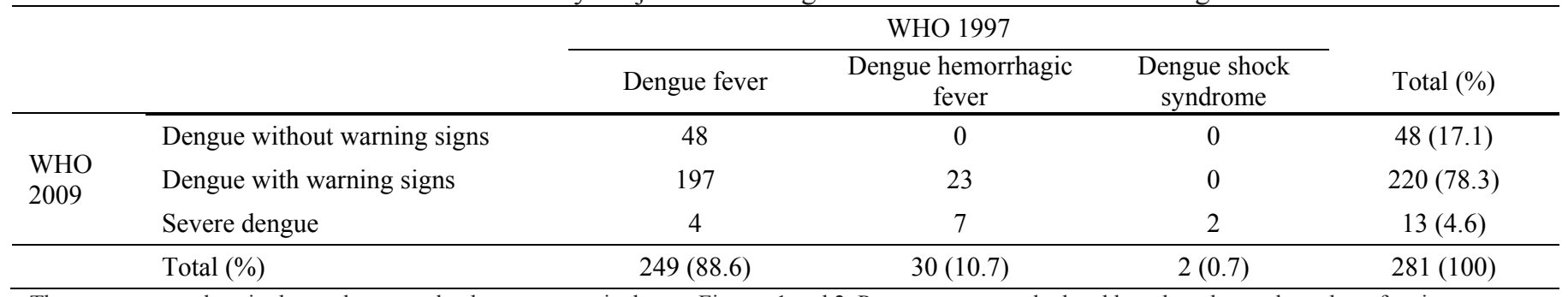

The percentage values in the total rows and columns are equivalent to Figures 1 and 2. Percentages are calculated based on the total number of patients. 


\section{Discussion}

Several studies $[10,13,14]$ reported that the revised WHO classification is more sensitive as well as more or comparably specific in identifying severe cases. In contrast, some still indicate that WHO 1997 is more sensitive - though less specific - in capturing severe cases [15].

We performed a retrospective observational study of dengue, which included 281 confirmed adult dengue patients (male: female ratio 1.5:1, range of age 13-88 years) admitted to the HUSM and HKB (between 2008 and 2010) as well as HSB (between 2010 and 2012).

We noted that the application of the two guidelines yielded different results. When classified according to WHO 1997 guidelines, our study cohort showed the highest percentage of DF, followed by DHF and DSS. This is in agreement with earlier reports $[14,16,17]$. However, the trend was different when the revised guidelines were used to classify the subjects. The highest percentage of cases were found to be DW, followed by D and SD. This finding was in line with that of Barniol et al. [10], whose study population included Malaysia. Although there are studies utilizing the 2009 guidelines $[14,16]$, their findings are not similar to each other and are different from those of this study as well.

Our results show that DW was the most common classification $(78.3 \%)$, followed by D (17.1\%) and SD (4.6\%). Narvaez et al. [14] found rates of both DW and SD to be similar and high in percentage (48\% and $44 \%$, respectively). Gan et al. [16] found D to be the most frequently occurring classification (48\%), followed by DW (36\%) and SD (16\%). These differences could be due to a very large number of samples [16] and the use of a pediatric cohort [14].

In the current study, WHO 2009 captured higher cases of SD than those captured as DSS by WHO 1997. Some of these discrepancies were explained by patients who had increased AST or ALT > 1000U/L. This deranged liver function has also been seen during dengue infection in many populations [18-20]. However, these patients had been classified as DF or DHF under the 1997 guidelines.

The low percentage of DHF in comparison to DW is expected, as DHF requires the patients to meet all four criteria. DW has looser categories, which allows us to capture more patients potentially at risk of developing severe manifestations. This may eventually prompt clinicians to treat patients aggressively before they progress into SD. Our high percentage of hospitalized patients classified as DW is in line with the recommendation of the WHO 2009 guidelines that patients with warning signs are to be referred for inpatient management. However, this may increase the burden on the health care system.

Several limitations were encountered in this study. First, dengue infection was only confirmed by clinical diagnosis followed by dengue-specific IgG and IgM serological tests, as these are the routine practice in the hospitals where the sampling was done. However, these tests were independently repeated at least twice to confirm the results. Diagnosis based on a single laboratory confirmation through dengue-specific IgM serological tests have also been applied elsewhere $[21,22]$. Second, different clinicians may classify any clinical manifestations of dengue fever differently. Third, to avoid any potential sampling biases, we did a similar analysis separately on the two cohorts (the 2008-2010 cohort versus the 2010-2012 cohort) and found no significant different between them (data not shown). Therefore, we believe that the impact of such limitations is minimal.

\section{Conclusions}

To the best of our knowledge, we have provided a platform of pros and cons for both new and old dengue classifications with the data from a Malaysian cohort. The revised 2009 WHO classification stratifies a much larger proportion of patients into a category that requires a higher level of medical and nursing care. This would have a significant impact on hospital resources in the region and would require a heightened level of awareness among health care personnel about the dangers of dengue.

\section{Acknowledgements}

We would like to thank all the clinicians and nurses as well as the recruited subjects from Hospital Universiti Sains Malaysia, Hospital Raja Perempuan Zainab II, and Hospital Sungai Buloh for their contributions to this study and Mrs. Suhaila Muid for her advice in the statistical analysis. This study was partly funded by FRGS 2007 (1001/PPSP/812039), FRGS 2010 [600 - RMI/ ST/FRGS 5/3/Fst (69/2010)], DANA Kecemerlangan UiTM [600 RMI/ STDANA 5/3/Dst (281/2009)] and the Long Term Research Grant Scheme (LRGS) for dengue research [600RMI/LRGS 5/3 (3/2011)].

\section{References}

1. World Health Organization (2009) Dengue: Guidelines for diagnosis, treatment, prevention and control. New edition. Geneva: World Health Organization.

2. Bhatt S, Gething PW, Brady OJ, Messina JP, Farlow AW, Moyes CL, Drake JM, Brownstein JS, Hoen AG, Sankoh O, Myers MF, George DB, Jaenisch T, Wint GR, Simmons CP, 
Scott TW, Farrar JJ, Hay SI (2013) The global distribution and burden of dengue. Nature 496: 504-507.

3. Ministry of Health Malaysia (2008) Clinical Practice Guideline: Management of Dengue Infection in Adult. Second edition.

4. Abubakar S, Shafee N (2002) Outlook of dengue in Malaysia: a century later. Malays J Pathol 24: 23-27.

5. World Health Organization (1997) Dengue haemorrhagic fever: diagnosis, treatment, surveillance, prevention and control, second edition. Geneva: World Health Organization.

6. Balmaseda A, Hammond SN, Perez MA, Cuadra R, Solano S, Rocha J, Idiaquez W, Harris E (2005) Short report: assessment of the World Health Organization scheme for classification of dengue severity in Nicaragua. Am J Trop Med Hyg 73: 1059-1062.

7. Gupta P, Khare V, Tripathi S, Nag VL, Kumar R, Khan MY, Dhole TK (2010) Assessment of World Health Organization definition of dengue hemorrhagic fever in North India. J Infect Dev Ctries 4: 150-155. doi:10.3855/jidc.708.

8. Ibrahim F, Guan CC, Sulaiman S, Taib MN, Wan Abas WA (2007) A new approach to classify risk in dengue infection using bioelectrical impedance analysis. Dengue Bulletin 31: 58-74.

9. Breen E, Pemmulu I, Ong DSH, Sekaran SD, Appana P, Tew TH, Khoo EM, Pillans L, Ismail NA, Omar SFS, Ponnampalavanar S, Vhimaleshwari R, Lum LCS (2012) Early predictors of dengue infection in adults (EPOD) - a Malaysian outpatient experience. Dengue Bulletin 36: 105115.

10. Barniol J, Gaczkowski R, Barbato EV, da Cunha RV, Salgado D, Martinez E, Segarra CS, Pleites Sandoval EB, Mishra A, Laksono IS, Lum LC, Martínez JG, Núnez A, Balsameda A, Allende I, Ramírez G, Dimaano E, Thomacheck K, Akbar NA, Ooi EE, Villegas E, Hien TT, Farrar J, Horstick O, Kroeger A, Jaenisch T (2011) Usefulness and applicability of the revised dengue case classification by disease: multi-centre study in 18 countries. BMC Infect Dis 11: 106.

11. Leo YS, Thein TL, Fisher DA, Low JG, Oh HM, Narayanan RL, Gan VC, Lye DC (2011) Confirmed adult dengue deaths in Singapore: 5-year multi-center retrospective study. BMC Infect Dis 11: 123.

12. Low JG, Ong A, Tan LK, Chaterji S, Chow A, Lim WY, Lee KW, Chua R, Chua CR, Tan SW, Cheung YB, Hibberd ML, Vasudevan SG, Ng LC, Leo YS, Ooi EE (2011) The early clinical features of dengue in adults: challenges for early clinical diagnosis. PLoS Negl Trop Dis 5: e1191.

13. Alexander N, Balmaseda A, Coelho IC, Dimaano E, Hien TT, Hung NT, Janisch T, Kroeger A, Lum LC, Martinez E, Siqueira JB, Thuy TT, Villalobos I, Villegas E, Wills B; European Union, World Organization (WHO-TDR) supported DENCO Study Group (2011) Multicentre prospective study on dengue classification in four South-east Asian and three Latin American countries. Trop Med Int Health 16: 936-948.
14. Narvaez F, Gutierrez G, Pérez MA, Elizondo D, Nuñez A, Balmaseda A, Harris E (2011) Evaluation of the traditional and revised WHO classifications of Dengue disease severity. PLoS Neg1 Trop Dis 5: e1397.

15. van de Weg CA, van Gorp EC, Supriatna M, Soemantri A, Osterhaus AD, Martina BE (2012) Evaluation of the 2009 WHO dengue case classification in an Indonesian pediatric cohort. Am J Trop Med Hyg 86: 166-170.

16. Gan VC, Lye DC, Thein TL, Dimatatac F, Tan AS, Leo YS (2013) Implications of discordance in world health organization 1997 and 2009 dengue classifications in adult dengue. PLoS One 8: e60946.

17. Srikiatkhachorn A, Gibbons RV, Green S, Libraty DH, Thomas SJ, Endy TP, Vaughn DW, Nisalak A, Ennis FA, Rothman AL, Nimmannitaya S, Kalayanarooj S (2010) Dengue hemorrhagic fever: the sensitivity and specificity of the world health organization definition for identification of severe cases of dengue in Thailand, 1994-2005. Clin Infect Dis 2010 50: 1135-1143.

18. Parkash O, Almas A, Jafri SM, Hamid S, Akhtar J, Alishah H (2010) Severity of acute hepatitis and its outcome in patients with dengue fever in a tertiary care hospital Karachi, Pakistan (South Asia). BMC Gastroenterol 10: 43.

19. Souza LJ, Alves JG, Nogueira RM, Gicovate Neto C, Bastos DA, Siqueira EW, Souto Filho JT, Cezário Tde A, Soares CE, Carneiro RC (2004) Aminotransferase changes and acute hepatitis in patients with dengue fever: analysis of 1,585 cases. Braz J Infect Dis 8: 156-163.

20. Wong M, Shen E (2008) The utility of liver function tests in dengue. Ann Acad Med Singapore 37: 82-83.

21. Khan E, Kisat M, Khan N, Nasir A, Ayub S, Hasan R (2010) Demographic and clinical features of dengue fever in Pakistan from 2003-2007: a retrospective cross-sectional study. PLOS One 5: e12505.

22. Karoli R, Fatima J, Siddiqi Z, Kazmi KI, Sultania AR (2012) Clinical profile of dengue infection at a teaching hospital in North India. J Infect Dev Ctries 6: 551-554. doi:10.3855/jidc. 2010 .

\section{Corresponding author}

Boon Peng Hoh

Institute of Medical Molecular Biotechnology (IMMB)

Faculty of Medicine, Universiti Teknologi MARA, Jalan Hospital 47000 Sungai Buloh, Selangor, Malaysia

Phone: 006-0361265000 ext: 7363

Fax: 006-0361265082

Email: hbpeng@salam.uitm.edu.my

Conflict of interests: No conflict of interests is declared. 